\title{
Temporal avoidance conditioning in the rabbit
}

\author{
RUSSELL A. LOCKHART AND DAVID G. STEINBRECHER \\ UNIVERSITY OF CALIFORNIA, SANTA BARBARA
}

\begin{abstract}
Abstraet
Rabbits were run in a temporal avoidance paradigm in which regularly occurring shock could be avoided only by jumping a barrier a few seconds prior to shock. Successful temporal avoidance conditioning was obtained, but the phenomenon, at least for the rabbit, is difficult to establish and subject to large individual differences.
\end{abstract}

\section{Problem}

Sidman $(1953,1956)$ has described an operant procedure for the development of avoidance behavior in a situation lacking an exteroceptive warning signal. In this procedure, a contingency exists between a response and the receipt of shock such that each response (e.g., cage turning) postpones a shock by $20 \mathrm{sec}$. Early in training, interresponse times vary considerably in which case the temporal intervals between shocks received is irregular. Thus, this procedure is essentially different from the temporal conditioning procedure originally reported by Pavlov (1927) in which the temporal interval between UCSs is constant. However, in the latter case the temporal CR is not instrumental in avoiding the UCS. It is reasonable, therefore, to determine whether or not it is possible to utilize the more or less classical conditioning approach in temporally conditioning an avoidance response. To this end, an experiment was done in which animals (rabbits) received an electric shock every 50 sec. unless a response (shuttle-box barrier crossing) occurred within a short period just prior to the shock (10-15 sec.). No other barrier crossings during the intershock interval were instrumental in avoiding the shock.

\section{Method}

New Zealand albino rabbits were selected as Ss for two reasons: (1) they are among the fastest learners of a shuttle-box avoidance response, and (2) their rate of spontaneous responding remains very low (Flakus \& Steinbrecher, 1964). Seven naive males about 3 mos. old were trained in a $4 \times 1 \times 1-1 / 2 \mathrm{ft}$ all black grid box with a 4-in barrier in the center. Either side of the grid floor could be charged with up to $40 \mathrm{ma}$ dc current provided by a Grason-Stadler shock generator (model E6070B). The shuttle box was located in a sound reduction room with a background illumination provided by 12 12-w fluorscent lights. Under these conditions there were no apparent external cues available to the animals. Animals were viewed through a one-way mirror and $E$ recorded the number as well as the time of occurrence of each barrier crossing.
$S$ was placed in the shuttle box and shocked every 50 sec. The current remained on until S escaped by jumping the barrier. Jump latency was about $1 \mathrm{sec}$. on the average. Shock could be avoided only if the rabbit jumped within a predetermined time period just prior to shock onset.

Each 50-sec. interval was viewed as one trial independently of S's behavior. Trials were divided into equal time periods each being as long as the avoidance interval. For each trial the number of spontaneous jumps occurring during these time periods were compared with the number of responses made during the avoidance period. Number of daily sessions, trials per session and length of avoidance period for each $\mathrm{S}$ are shown in Table 1.

\section{Results}

The basic data consist of the number of responses made during the avoidance interval and the number of responses made during a "comparison" or "control" interval of equal duration preceding the avoidance interval. Presumably, if temporally conditioned avoidance responding occurs the number of responses in the former interval should exceed that in the latter interval.

Table 2 presents the mean number of response during the avoidance interval as well as the control interval over all trials for each S.

For each S a Wilcoxon matched-pairs signed-rarks test was employed to determine if the number of responses during the avoidance interval exceeded that in the control interval. For five of the seven animals the difference was highly significant. When considered as a group, the difference between avoidance and comparison responses was also highly significant $(\mathrm{T}=0$; $\mathrm{N}=7 ; \mathrm{p}<.01$ ).

Figure 1 presents the mean percent of temporal avoidance (defined as the difference in percent of jumps between the test and control values) for each

TABLE I

Number of Daily Training Sessions, Trials per Session and Duration of Avoidance Period for each $\mathbf{S}$

\begin{tabular}{cccc} 
S & $\begin{array}{c}\text { Number of } \\
\text { sessions }\end{array}$ & $\begin{array}{c}\text { Trials } \\
\text { per session }\end{array}$ & $\begin{array}{c}\text { Avoidance } \\
\text { interval (sec.) }\end{array}$ \\
\hline 1 & 40 & 20 & 10 \\
8 & 20 & 10 & 15 \\
9 & 20 & 10 & 15 \\
10 & 40 & 10 & 15 \\
11 & 40 & 10 & 15 \\
12 & 30 & 10 & 15 \\
13 & 30 & 10 & 15
\end{tabular}


animal for each fifth of acquisition. Curves on the left of the figure are for animals having positive differences at each point. Those on the right are characterized by one or more negative differences (i.e., greater response frequency in the control than in the test interval) during acquisition.

\section{Diseussion}

The data provide sufficient evidence for a phenomenon that might be termed temporal avoidance conditioning. Five of seven animals jumped significantly more during a period which led to shock avoidance than during a period which did not affect receipt of shock. It should be noted, however, that the phenomenon is difficult to establish and for some animals seems quite evanescent. Only two animals showed reliable avoidance responding (Ss 1 and 13) reaching 80 to $90 \%$ within a session at various points during acquisition.

Difficulty in establishing an overt avoidance response in a situation without an exteroceptive signal may be related to phyletic level. For this reason, a similar attempt will be made with cats. In addition, it seems important to gain information on automatic responding during the learning of this task. Dykman, Gantt, \& Whitehorn (1956), among others, have reported that temporally conditioned skeletal behavior appears subsequent to temporally conditioned autonomic responses.

Finally, the rather large degree of individual differences between the Ss should not go unnoticed. There seem to exist three different response patterns. Animals 1 and 13 developed a quite high rate of responding

TABLE 2

Mean Number of Jumps in Avoidance and Control Intervals for each $\mathbf{S}$

\begin{tabular}{|c|c|c|c|}
\hline \multirow[t]{2}{*}{$\mathrm{S}$} & \multicolumn{2}{|c|}{ Mean Number of Jumps } & \multirow[b]{2}{*}{ Difference } \\
\hline & Avoidance & Control & \\
\hline 1 & 7.85 & 3.45 & $4.40 \star \star \star$ \\
\hline 8 & 10.05 & 10.00 & .05 \\
\hline 9 & 0.95 & 0.70 & .25 \\
\hline 10 & 4.75 & 3.60 & $1.15^{\star \star}$ \\
\hline 11 & 7.13 & 6.03 & 1. $10 \star$ \\
\hline 12 & 12.87 & 12.53 & $.34^{\star}$ \\
\hline 13 & 3.63 & 0.90 & $2.73^{\star \star \star}$ \\
\hline
\end{tabular}

${ }^{*} p<.0003 \quad{ }^{*} \mathrm{p}<.00005 \quad * \star * \mathrm{p}<.00003$

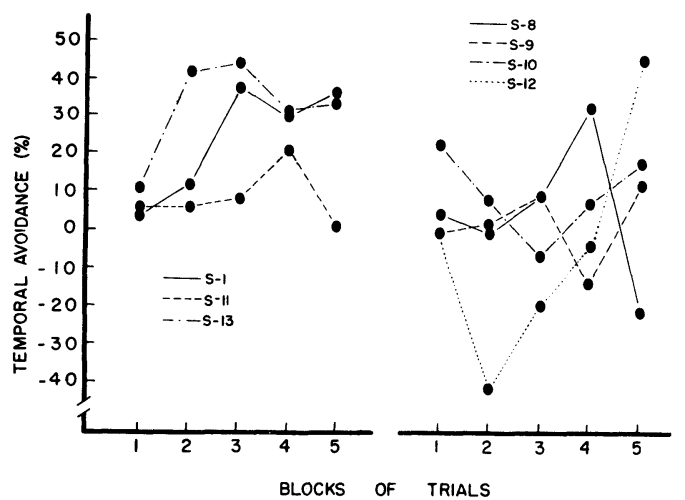

Fig. 1. Mean temporal a voidance $(\%)$ for each fifth of acquisition for each S. (Ss 2-7 were run under different experimental conditions than reported here.)

in the avoidance interval with very little responding during the other intervals. In these animals "temporal discrimination" was quite evident. Animals 8 and 12 developed a "jumping syndrome", i.e., they would jump a large number of times during the entire trial interval. These Ss avoided almost every shock but did not develop a differential jumping pattern between the avoidance interval and the control interval. Animals 9,10 and 11 were characterized by very little jumping and consequently received the largest number of shocks. These animals developed a small, but consistant preference for jumping in the avoidance interval.

\section{Refereneen}

Flakus, W. J., \& Steinbrecher, D. C. Avoidance conditioning in the rabbit. Psychol. Rep., 1964, 14, 140.

Dykman, W. A., Gantt, W. H., \& Whitehom, J. C. Conditioning as emotional sensitization and differentiation. Psychol. Monogr., 1956, 70, No. 422 .

Sidman, M. Two temporal parameters of the maintenance of avoidance behavior in the white rat. J. comp. physiol. Psychol., $1953,46,253-261$.

Sidman, M. Avoidance conditioning with brief shock and no exteroceptive warning signal. Science, 1956, 118, 157-158.

\section{Note}

1. This investigation was supported in part by Public Health Service Grant MH 11723-01 from the National Institute of Mental Health. 